\title{
Morphological Lesion Types Are Associated with Primary and Secondary Patency Rates after High- Pressure Balloon Angioplasty for Dysfunctional Arteriovenous Fistulas
}

\author{
Li Chen $^{\text {a }}$ Weichen Zhang ${ }^{b}$ Jinyun Tan ${ }^{c}$ Min Huc Weihao Shi ${ }^{d}$ \\ Minmin Zhang ${ }^{b}$ Yong Wang ${ }^{a}$ Bo $\mathrm{Yu}^{\mathrm{c}, \mathrm{d}}$ Jing Chen ${ }^{\mathrm{b}}$
}

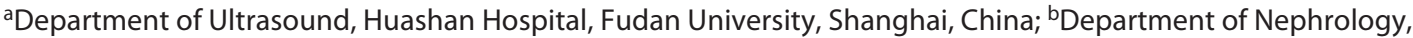
Huashan Hospital, Fudan University, Shanghai, China; 'Department of Vascular Surgery, Huashan Hospital, Fudan University, Shanghai, China; 'Department of Vascular Surgery, Pudong Hospital, Fudan University, Shanghai, China

\section{Keywords}

Hemodialysis · Arteriovenous fistula dysfunction ·

Neointimal hyperplasia $\cdot$ Morphological type $\cdot$ Patency rate

\begin{abstract}
Background: Neointimal hyperplasia $(\mathrm{NIH})$ is believed to be the main reason for arteriovenous fistula (AVF) dysfunction, but other mechanisms are also recognized to be involved in the pathophysiological process. This study investigated whether different morphological types of AVF lesions are associated with the patency rate after percutaneous transluminal angioplasty (PTA). Methods: This retrospective study included 120 patients who underwent PTA for autogenous AVF dysfunction. All the cases were evaluated under Doppler ultrasound (DU) before intervention and divided into 3 types: Type I (NIH type), Type II (non-NIH type), and Type III (mixed type). Prognostic and clinical data were analyzed by Kaplan-Meier analysis and the Cox proportional hazards model. Results: There was no statistical difference in baseline variables among groups, except for lumen diameter. The primary patency rates in Type I, Type II, and Type III groups were $78.4,93.2$, and $83.2 \%$ at 6 months and 59.5, 84.7, and $75.5 \%$ at 1 year, respectively. The secondary patency rates in
\end{abstract}

karger@karger.com www.karger.com/bpu

Karger $\stackrel{\text { ' }}{5}$
(C) 2021 The Author(s)

Published by S. Karger AG, Basel

This is an Open Access article licensed under the Creative Common Attribution-NonCommercial-4.0 International License (CC BY-NC) (http://www.karger.com/Services/OpenAccessLicense), applicable to the online version of the article only. Usage and distribution for commercial purposes requires written permission.
Type I, Type II, and Type III groups were 94.4, 97.1, and 100\% at 6 months and $90.5,97.1$, and $94.7 \%$ at 1 year, respectively. The Kaplan-Meier curve showed that the primary and secondary patency rates of Type I group were lower than those of Type II group. Multivariable Cox regression analysis demonstrated that postoperative primary patency was correlated with end-to-end anastomosis (hazard ratio $[\mathrm{HR}]=2.997$, $p=0.008,95 \%$ confidence interval [Cl]: 1.328-6.764) and Type I lesion ( $\mathrm{HR}=5.395, p=0.004,95 \% \mathrm{Cl}: 1.730-16.824)$. Conclusions: $\mathrm{NIH}$-dominant lesions of AVF evaluated by DU preoperatively were a risk factor for poor primary and secondary patency rate after PTA in hemodialysis patients.

(C) 2021 The Author(s).

Published by S. Karger AG, Basel

\section{Introduction}

In China, over 500,000 patients with end-stage renal disease (ESRD) rely on hemodialysis treatment, and $80.5-88.3 \%$ of those patients use an autogenous arteriovenous fistula $(\mathrm{AVF})[1,2]$. However, AVF stenosis is

Li Chen and Weichen Zhang contributed equally to this work.
Correspondence to:

Jinyun Tan, m.tan@fudan.edu.cn 
common and may lead to a series of problems, such as inadequate dialysis volume, difficulties in cannulation, and ultimately thrombotic events. Endovascular therapy has become a first-line treatment to correct stenosis of dysfunctional AVFs, but it needs to be repeated frequently to maintain AVF patency [3].

Neointimal hyperplasia (NIH) is the major reason for AVF stenosis; however, new evidence shows that besides $\mathrm{NIH}$, other mechanisms, such as adventitia constriction, valve-related stenosis, and inflow artery atherosclerosis, also participates in the pathological process [4-9]. Lesion features can be detected by Doppler ultrasound (DU), which is often undertaken before interventions for dysfunctional AVFs, but it is unclear whether certain morphological lesion types have a unique response to angioplasty treatment. As a consequence, physicians have difficulties selecting an individualized therapeutic strategy for these particular patients.

In this study, cases receiving endovascular repair for dysfunctional AVFs were classified into 3 types according to the preoperative DU imaging. We evaluated the primary and secondary patency rates after percutaneous transluminal angioplasty (PTA) with a high-pressure balloon to determine whether the morphological types of dysfunctional AVFs with clinical indicators are associated with the results of interventional repair.

\section{Materials and Methods}

\section{Patients and Data Collection}

This is a retrospective study conducted in a tertiary academic referral center and approved by the Ethics Committee on Human Research at Huashan Hospital, Fudan University, Shanghai (KY2017-429). From January 2015 to December 2018, 192 consecutive ESRD patients who received PTA for a dysfunctional AVF were reviewed (online suppl. Fig. 1; for all online suppl. material, see www.karger.com/doi/10.1159/000516883). The indication for PTA was an AVF with $>50 \%$ stenosis in conjunction with at least one clinical or physiological abnormality, such as inadequate dialysis flow and abnormal physical findings according to $2006 \mathrm{Kid}$ ney Disease Outcomes Quality Initiative guidelines [10]. In this cohort, we only performed PTA for patients who had been receiving hemodialysis for at least 1 month. PTA for immature AVFs was excluded. Hybrid procedures, adjacent drug-eluted balloon angioplasty, or stent implantation was also excluded, leaving 120 cases treated by standard high-pressure angioplasty to analyze. All patients provided written informed consent. The preoperative demographic data, procedure information, and follow-up details were all collected by electronic medical records.

Ultrasound Assessment and Stenosis Classification

Before the operation, each patient underwent preoperative measurements and vein mapping. The inflow arteries, anastomot- ic sites, and outflow veins were all scanned, and the intimal thickness, flow volume, and inner and outer diameters at the stenosis sites were recorded to identify the morphological patterns. The typical sonograms are shown in Figure 1. All the lesions were categorized as Types I, II, or III. In Type I group, stenosis was $>50 \%$, and the intimal thickness measured $>0.6 \mathrm{~mm}$ at the stenosis lesion site without concomitant inflow artery or distal vein stenosis, which can cause flow limitation. Type I is thought to be initially caused by NIH. In Type II group, the stenosis was $>50 \%$, and intimal thickness measured $\leq 0.6 \mathrm{~mm}$ at the stenosis lesion site without concomitant inflow artery or distal vein stenosis. NIH is not thought to be the initial cause of stenosis in Type II lesions. In Type III group, the stenosis was $>50 \%$ combined with tandem lesions, such as inflow artery, anastomosis, and outflow vein stenosis on preoperative ultrasonogram evaluation, regardless of the presence of intimal hyperplasia. This is thought to be a mixed type with an uncertain etiology but not rare in the real world. All the preoperative and follow-up ultrasonography were measured by linear transducer on DU scanners (including HITACHI HI VISION Preirus with a 5-13-MHz linear transducer EUP-L74, Philips IU-22 with a 5-17-MHz linear transducer L17-5, and Philips EPIQ5 with a 5-18-MHz linear transducer L18-5) and reviewed by an independent ultrasound specialist.

\section{Interventional Procedures}

All procedures were performed under guided DU. Under local anesthesia, a 5-F or 6-F vascular sheath (Terumo Inc., Japan) was inserted in the outflow vein toward the stenosis, and then semiheparinization was performed with 40-50 U/kg unfractionated heparin. A 0.035-in. guidewire (Terumo Inc., Japan) was introduced through the sheath and traversed the lesion under ultrasound guidance. In most cases, the tip of guidewire was delivered to the level of the proximal arteries (e.g., brachial artery) to ensure the stability of the working guidewire. According to the diameter of the veins and the length of the lesion, a noncompliant highpressure balloon (Mustang, Boston Scientific Inc., USA) with a diameter $1 \mathrm{~mm}$ greater than the normal diameter of the venous segment was introduced over the guidewire; the size of the balloons ranged from 4 to $7 \mathrm{~mm}$ in diameter, and they were $40 \mathrm{~mm}$ in length. During dilatation, the lesion was covered by the middle part of the balloon. The balloon was inflated to its rated burst pressure and maintained for at least 1 min until the balloon waist disappeared under the monitor of the DU. Remeasurement of the diameter and flow volume at the lesion site and the brachial artery were routinely performed prior to removal of the sheath. Technical success was defined as $<30 \%$ residual stenosis, $>500 \mathrm{~mL} / \mathrm{min}$ flow volume in the brachial artery, and perception of a continuous palpable thrill. For residual stenosis $>30 \%$, repeated angioplasty or dilation by a larger balloon would be performed; an adjunctive stent was used in the upper arm, while the flow-limiting recoil persisted.

\section{Pathologic Studies of Vascular Specimens}

The vascular lesion specimens were obtained from open fistula reconstruction surgery cases to confirm the consistency between ultrasonographic and pathological studies. The indication for open surgery repair was failed reintervention by endovascular procedure or restenosis/occlusion in $<3$ months. Cross sections of AVF samples were fixed in $4 \%$ neutral-buffered paraformaldehyde and processed for light microscopy. All tissue samples were stained 

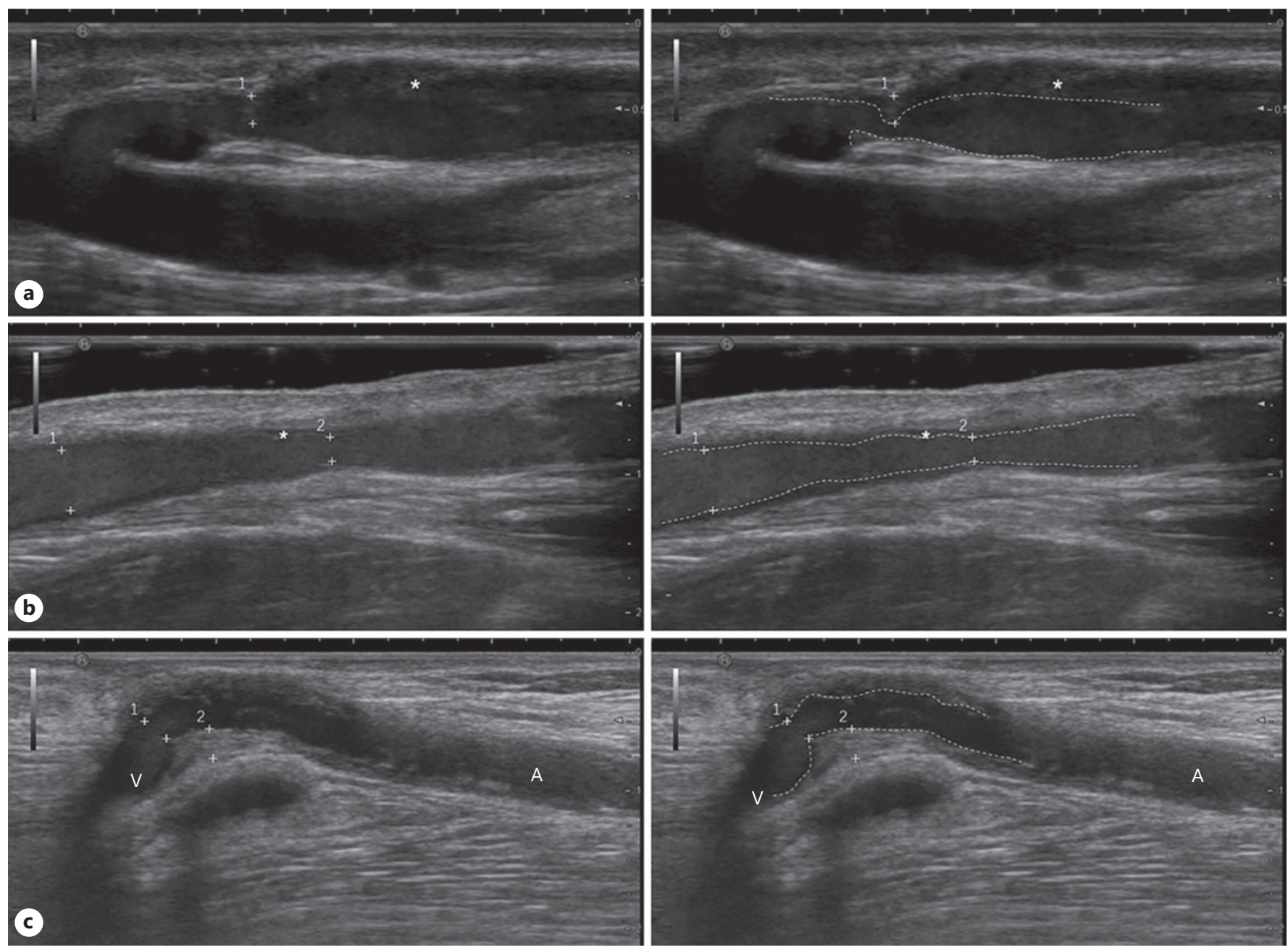

Fig. 1. Ultrasonograms of typical AVF lesions. a Type I, intimal hyperplasia at the proximal vein: distance 1 shows the thickness of intima $(1.5 \mathrm{~mm})$; the residual lumen is $0.8 \mathrm{~mm}$. b Type II, conduit constriction at the vein: distance 1 and 2, respectively, represent the normal $(4.4 \mathrm{~mm})$ and constricted lumen $(1.7 \mathrm{~mm})$; the intima

$\left.{ }^{*}\right)$ is $<0.3 \mathrm{~mm}$. c Type III, plaque at the artery: distance 1 and 2 represent the bypass $(2.0 \mathrm{~mm})$ and the thickness of plaque $(2.0$ $\mathrm{mm})$, respectively. The borders between intima and lumen are shown on the figure as dotted lines. + measure; * intima; A, artery; $\mathrm{V}$, vein; AVF, arteriovenous fistula.

with hematoxylin and eosin and Masson's trichrome stain to identify the relative thickness of the intima and media $[8,11]$. All histologic evaluations were performed by a single pathologist without clinical characteristics of the study patients and AVF outcomes.

\section{Follow-Up and Outcome Definitions}

After the procedure, patients were transferred to a dialysis center and followed up by the attending nephrologists before each dialysis session and evaluated by vascular surgeons at 3, 6, 12, 18, and 24 months after the initial operation. During the follow-up vintage, primary patency was defined as the interval that a patent fistula maintained continued success and efficient dialysis sessions without the need for repeat endovascular therapy or surgical revision, while secondary patency was defined as the time from the repairing operation until access abandonment, which was not ter-

minated by interventional procedures to maintain or restore patency.

\section{Statistical Analysis}

Quantitative variables of patients' characteristics are expressed as mean \pm standard deviation or as median (interquartile range), while categorical variables are expressed as percentages or ratios. Differences were evaluated using $\chi^{2}$ test and independent $t$ test. The patency rate was estimated using the Kaplan-Meier method, while intergroup comparisons were assessed with the log-rank test. A Cox proportional hazards model was used to identify independent factors associated with primary and secondary patency after adjustment for confounders. Statistical analysis was performed using SPSS version 19 (Chicago, IL, USA). A $p$ value $<0.05$ was considered statistically significant. 
Table 1. Baseline characteristics of patients of overall, Type I, Type II, and Type III

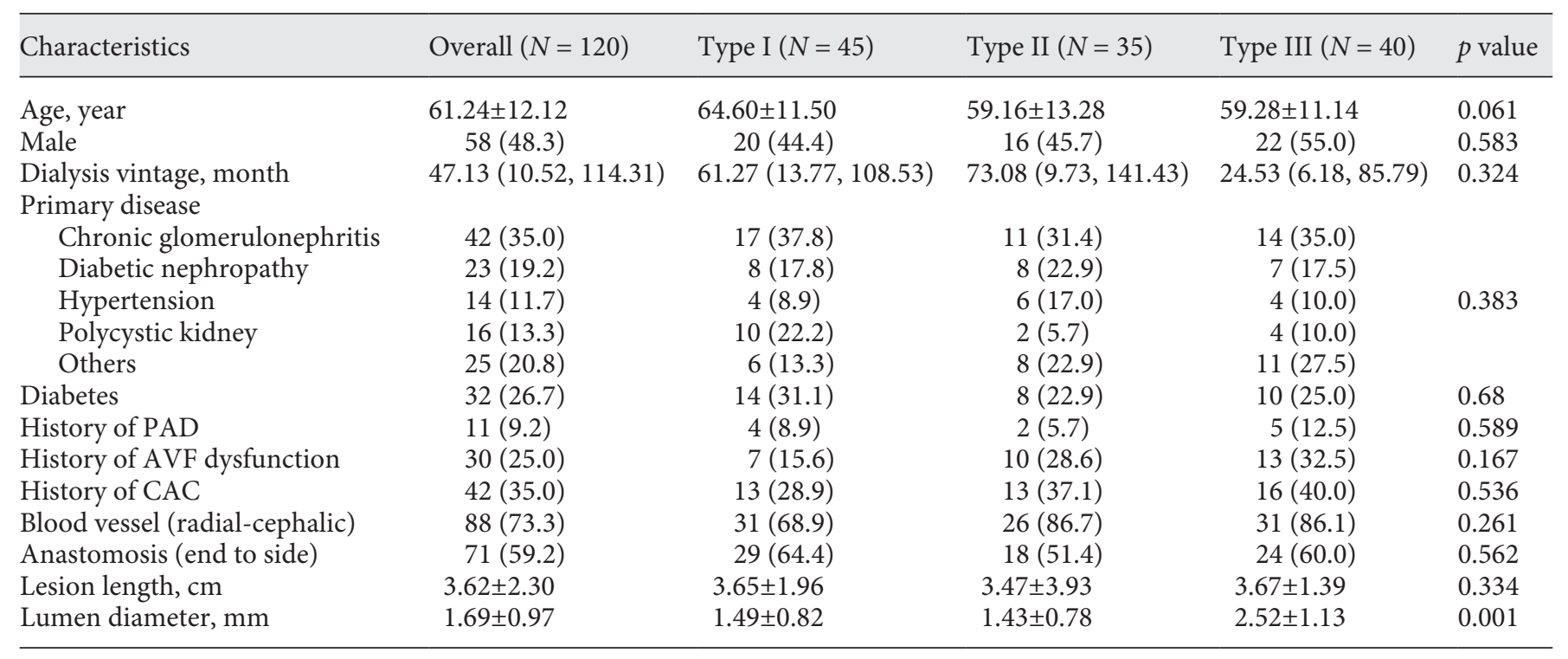

$\mathrm{IH}$, intimal hyperplasia; AVF, arteriovenous fistula; PAD, peripheral arteria disease; CAC, central venous catheterization; Type I, IH-dominant lesion type; Type II, non-IH lesion type; Type III, partial-IH lesion type; SD, standard deviation; IQR, interquartile range. Data are expressed as mean $\pm \mathrm{SD}$ or median (IQR) or number (percentage).

\section{Results}

Table 1 describes the demographic, clinical, and vascular access characteristics of the 3 groups and of the full cohort of 120 patients. The mean age of the entire patient cohort was $61.2 \pm 12.1$ year, and $48.3 \%$ were male. The median dialysis vintage was 47.1 months. The primary causes of ESRD included chronic glomerulonephritis (35.0\%), diabetic nephropathy (19.2\%), hypertension (11.7\%), polycystic kidney disease (13.3\%), and other diseases (20.8\%). Fistulas served a median vintage of 24.6 months before intervention, and $74.2 \%$ of AVFs were created on the left arm. Type I lesions were observed in 45 (37.5\%) patients, while Types II and III included 35 (29.2\%) and 40 (33.3\%) patients, respectively. There was no significant difference among the 3 groups in baseline characteristics, except for the lumen diameter, which was the largest in Type III group.

A total of 26 patients in this cohort underwent open fistula reconstruction surgery during the follow-up. Vascular samples obtained during the procedure underwent pathological examination (Fig. 2); 8 cases diagnosed as Type I lesions were characterized by neointimal proliferation and 6 diagnosed as Type II featured adventitia constriction (no valve-related lesions in these cases). All pathological diagnoses were consistent with the morphological evaluation by ultrasonography.
In this study, the primary patency rates in Type I, Type II, and Type III groups were $78.4,93.2$, and $83.2 \%$ at 6 months and 59.5, 84.7, and $75.5 \%$ at 1 year, respectively. The association between AVF stenosis type and AVF primary patency was analyzed using a Kaplan-Meier curve (Fig. 3) and Cox regression (Table 2). The Kaplan-Meier curve showed that the non-NIH group had the highest primary patency among 3 groups $(p=0.001)$. In detail, the primary patency was higher in Type II group than in Type III group ( $p=0.015$ ), but there was no significant difference between Type I and Type III groups ( $p=$ 0.216). Multiple Cox regression analysis demonstrated that the postoperative AVF primary patency was correlated with end-to-end anastomosis (hazard ratio [HR] = $2.997, p=0.008$, and $95 \%$ confidence interval $[\mathrm{CI}]: 1.328$ $6.764)$ and AVF lesion type ( $p=0.012$ ).

The secondary patency rate was $97.0 \%$ of the whole cohort at 6 months and $94.2 \%$ at 12 months. Per group, the 6-month and 1-year secondary patency rates were 94.4 and $90.5 \%$ in Type I group, 97.1 and $97.1 \%$ in Type II group, and 100 and $94.7 \%$ in Type III group. Univariable and multivariable analysis results of the association between AVF stenosis type and AVF secondary patency are shown in Figure 3 and Table 3. The Kaplan-Meier curve showed that the secondary patency of Type I group was less than that of Type II and Type III groups $(p=0.003)$, while there was no 

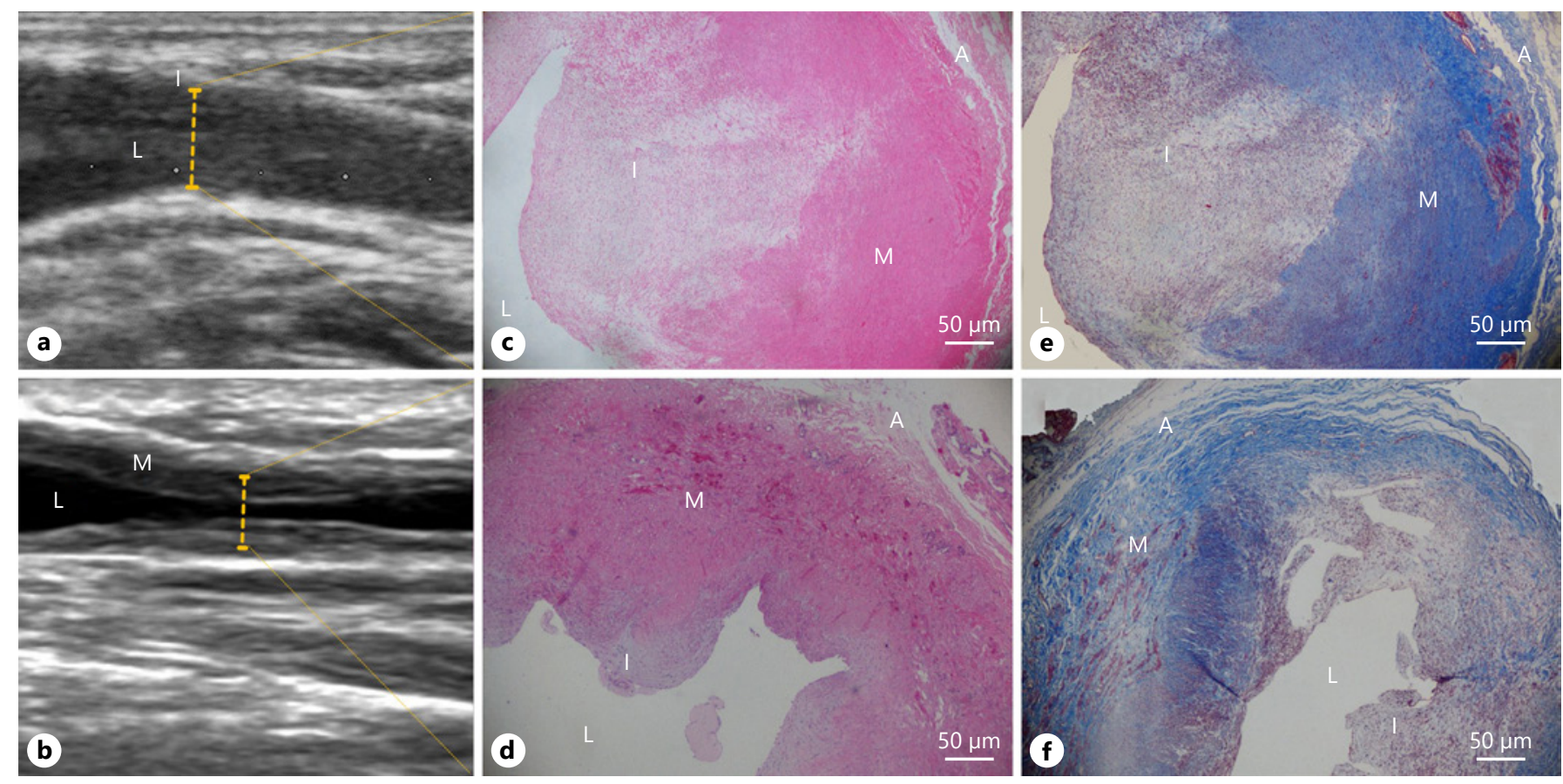

Fig. 2. Ultrasonic and histopathological images of AVF lesions. Ultrasonic images show an AVF with significant Type I lesion (a) and an AVF with significant Type II lesion (b). HE stain (c, d) and Masson's trichrome stain (e, f) of these samples show their respective histopathology. L, lumen; I, intima; M, media; A, adventitia; AVF, arteriovenous fistula; HE, hematoxylin and eosin.

Table 2. Cox regression analysis of primary patency after PTA of AVF reconstruction

\begin{tabular}{|c|c|c|c|c|c|}
\hline \multirow[t]{2}{*}{ Characteristics } & \multicolumn{2}{|c|}{ Univariable Cox regression } & \multicolumn{3}{|c|}{ Multivariable Cox regression } \\
\hline & HR & $p$ value & HR & $p$ value & $95 \% \mathrm{CI}$ \\
\hline Age, year & 1.015 & 0.277 & 1.007 & 0.659 & $0.975-1.041$ \\
\hline Male & 0.7 & 0.297 & 0.473 & 0.062 & $0.215-1.039$ \\
\hline Dialysis vintage, month & 1.001 & 0.956 & & & \\
\hline Primary disease & - & 0.72 & & & \\
\hline Diabetes & 1.79 & 0.101 & & & \\
\hline History of PAD & 2.095 & 0.126 & & & \\
\hline History of AVF dysfunction & 1.096 & 0.796 & & & \\
\hline History of CAC & 0.849 & 0.635 & & & \\
\hline Blood vessel (radial-cephalic) & 0.486 & 0.069 & & & \\
\hline Anastomosis (end to end) & 3.022 & 0.007 & 2.997 & 0.008 & $1.328-6.764$ \\
\hline Lesion type & - & 0.003 & - & 0.012 & - \\
\hline Type I & 4.932 & 0.002 & 5.395 & 0.004 & $1.730-16.824$ \\
\hline Type II & - & Ref & - & Ref & - \\
\hline Type III & 2.083 & 0.175 & 3.135 & 0.073 & $0.900-10.916$ \\
\hline Lesion length, $\mathrm{cm}$ & 1.005 & 0.961 & & & \\
\hline Lumen diameter, $\mathrm{mm}$ & 0.885 & 0.556 & & & \\
\hline
\end{tabular}

PTA, percutaneous angioplasty; AVF, arteriovenous fistula; PAD, peripheral arteria disease; CAC, central venous catheterization; HR, hazard ratio; $95 \% \mathrm{CI}$, 95\% confidence interval; Ref, reference; HR = 1.0; Type I, IHdominant lesion type; Type II, non-IH lesion type; Type III, partial-IH lesion type. 
Fig. 3. Kaplan-Meier curve of primary (a) and secondary patency $(\mathbf{b})$ of patients with Type I, Type II, and Type III lesions.

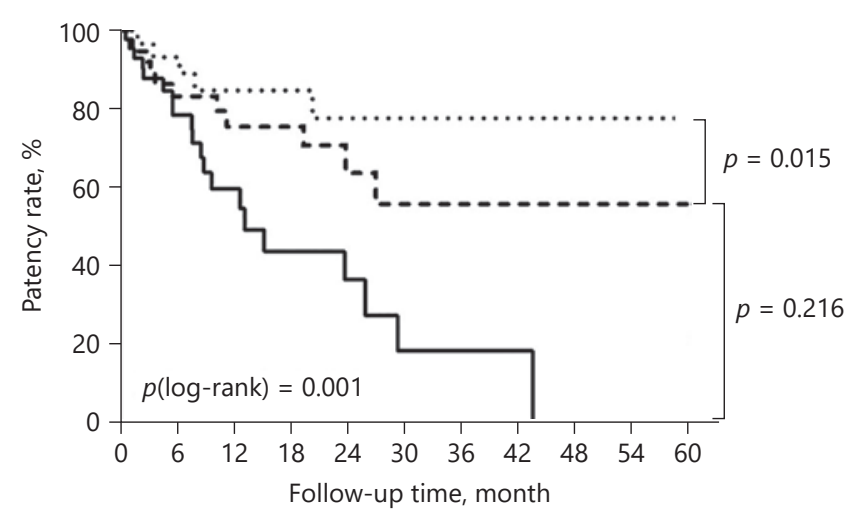

- Type I

..... Type ॥

- - - Type III

\begin{tabular}{|c|c|cccc|}
\hline \multicolumn{2}{|c|}{ Group } & \multicolumn{4}{c|}{ Month } \\
\cline { 3 - 6 } & Number at risk & 45 & 6 & 12 & 24 \\
\hline \multirow{2}{*}{ Type I } & Number of events & 0 & 24 & 12 & 5 \\
& Number at risk & 35 & 8 & 13 & 17 \\
\hline \multirow{2}{*}{ Type II } & Number of events & 0 & 25 & 15 & 11 \\
& Number at risk & 40 & 2 & 4 & 5 \\
\hline \multirow{2}{*}{ Type III } & Number of events & 0 & 6 & 18 & 9 \\
& & & 26 & 8 & 10 \\
\hline
\end{tabular}

a

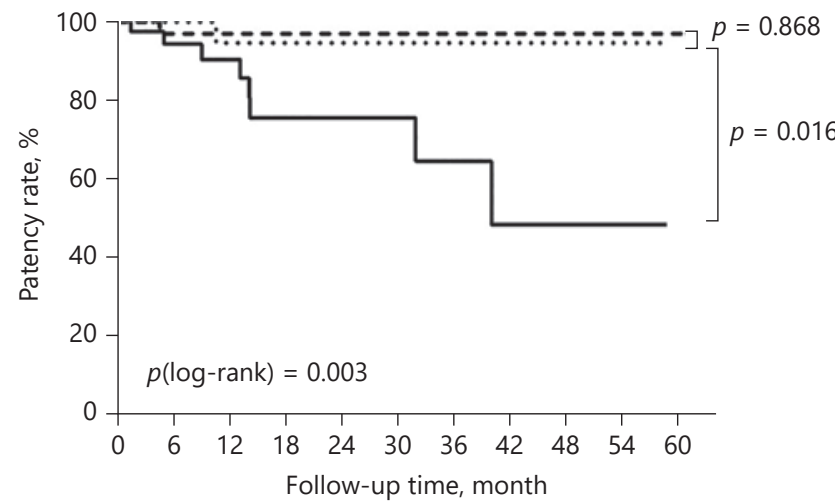

\begin{tabular}{|c|c|cccc|}
\hline \multicolumn{2}{|c|}{ Group } & \multicolumn{4}{c|}{ Month } \\
\cline { 3 - 6 } & Number at risk & 45 & 6 & 12 & 24 \\
\hline \multirow{2}{*}{ Type I } & Number of events & 0 & 28 & 19 & 9 \\
& Number at risk & 35 & 2 & 3 & 6 \\
\hline \multirow{2}{*}{ Type II } & Number of events & 0 & 25 & 17 & 14 \\
& Number at risk & 40 & 31 & 1 & 1 \\
\hline \multirow{2}{*}{ Type III } & Number of events & 0 & 1 & 1 & 15 \\
& & &
\end{tabular}

b

significant difference between Type II and Type III groups $(p=0.868)$. Multiple Cox regression analysis demonstrated that the postoperative AVF secondary patency was correlated with the history of AVF dysfunction (HR = 16.741, $p=0.009$, and 95\% CI: 2.009-139.525), end-to-end anastomosis (HR $=16.117, p=0.006$, and 95\% CI: 3.214-114.193), and AVF lesion type $(p=0.017)$.

\section{Discussion}

Our study demonstrated that morphological features in dysfunctional AVFs are associated with the primary and secondary patency rate after PTA with a high-pressure balloon. In the 2019 update of Kidney Disease Outcomes Quality Initiative guidelines for vascular access, 
Table 3. Cox regression analysis of secondary patency after PTA of AVF reconstruction

\begin{tabular}{|c|c|c|c|c|c|}
\hline \multirow[t]{2}{*}{ Characteristics } & \multicolumn{2}{|c|}{ Univariable Cox regression } & \multicolumn{3}{|c|}{ Multivariable Cox regression } \\
\hline & $\mathrm{HR}$ & $p$ value & $\mathrm{HR}$ & $p$ value & $95 \% \mathrm{CI}$ \\
\hline Age, year & 0.999 & 0.958 & 0.946 & 0.234 & $0.864-1.036$ \\
\hline Male & 0.52 & 0.343 & 0.254 & 0.134 & $0.042-1.526$ \\
\hline Dialysis vintage, month & 0.998 & 0.805 & & & \\
\hline Primary disease & - & 0.474 & & & \\
\hline Diabetes & 1.641 & 0.474 & & & \\
\hline History of PAD & 3.404 & 0.222 & & & \\
\hline History of AVF dysfunction & 4.447 & 0.068 & 16.741 & 0.009 & $2.009-139.525$ \\
\hline History of CAC & 0.577 & 0.428 & & & \\
\hline Blood vessel (radial-cephalic) & 0.166 & 0.25 & & & \\
\hline Anastomosis (end to end) & 9.873 & 0.032 & 16.117 & 0.006 & $3.214-114.193$ \\
\hline Lesion type & - & 0.021 & - & 0.017 & - \\
\hline Type I & 8.354 & 0.046 & 34.67 & 0.013 & $2.130-65.406$ \\
\hline Type II & - & Ref & - & Ref & - \\
\hline Type III & 0.838 & 0.901 & 2.844 & 0.51 & $0.127-36.726$ \\
\hline Lesion length, cm & 0.948 & 0.815 & & & \\
\hline Lumen diameter, $\mathrm{mm}$ & 0.805 & 0.584 & & & \\
\hline
\end{tabular}

PTA, percutaneous angioplasty; AVF, arteriovenous fistula; PAD, peripheral arteria disease; CAC, central venous catheterization; HR, hazard ratio; $95 \% \mathrm{CI}$, 95\% confidence interval; Ref, reference; HR = 1.0; Type I, IHdominant lesion type; Type II, non-IH lesion type; Type III, partial-IH lesion type.

endovascular angioplasty (with a high-pressure balloon as needed) was considered a reasonable primary treatment for AVF stenotic lesions with clinical indicators [3]. PTA could immediately restore the flow for hemodialysis while conserving vascular resource for patients, but the high restenosis and reintervention rate remains a significant challenge $[12,13]$.

Neointimal proliferation is considered to be the main reason for AVF dysfunction by the authoritative textbooks of both nephrology and vascular surgery $[14,15]$. However, recent studies have demonstrated that pathological types other than NIH result in dysfunctional AVFs, such as vascular constriction caused by adventitia fibroblasts proliferation, arterial negative remodeling, or atherosclerosis. In 2014, Simone et al. [9] showed that adventitial remodeling played an important role in AVF stenosis and that an increase of adventitial fibrosis and myofibroblast activation was associated with AVF failure. Tabbara et al. [16] investigated biopsy specimens from a prospective cohort undergoing two-stage AVF creation and transportation, and they found that postoperative NIH was not linked to AVF failure or to the loss of primary unassisted patency and revealed no occlusive NIH from the AVF tissue obtained at surgery [7]. Rothuizen et al. [4] postulated that the balance between intimal hyperplasia and vascular outward remodeling may ultimately determine fistula flow and patency. Therefore, it is recognized that intimal hyperplasia does not account for all the stenosis lesions, and an effective method to evaluate and distinguish different patterns of lesions before treatment is warranted.

In this study, the NIH type accounted for $37.5 \%$ of all the dysfunctional AVFs. The primary patency rates in this group were $78.4,59.5$, and $36.4 \%$ at 6,12 , and 24 months, respectively; when compared with the non-NIH group's (29.2\% of all dysfunctional AVFs) rates of 93.2, 84.7, and $77.1 \%(p=0.001)$, these values reflect the discrepant prognosis between these 2 groups featured by differing severity of neointimal proliferation. The criteria of classification were identified by preoperative DU. The advantage of DU is its ability to detect the properties of the structure inside stenosis lesions, whereas digital subtraction angiography can only demonstrate intraluminal fluid information by contrast. The morphological features were significantly different between $\mathrm{NIH}$-dominant and non-NIH lesions. In our experience, the histopathology observation of stenosis lesion specimens taken from fistula repair surgery was highly consistent with the morphologic characteristics evaluated by DU.

To date, few studies have evaluated the impact of different types of AVF/arteriovenous graft (AVG) lesions on the patency rates after intervention. Yamamoto et al. [17] described a cohort of 46 AVG outflow stenosis cases 
whose lesions were divided into 3 groups by DU evaluation: vascular constriction types (13/46, 28.3\%), neointimal proliferation types $(12 / 46,26.1 \%)$, and mixed types $(21 / 46,45.7 \%)$; the vascular constriction type in their study displayed excellent primary patency rates after stent treatment. Suemitsu et al. [18] investigated 158 AVF venous lesions and divided them into 3 stenosis patterns by ultrasonography: intimal hyperplasia stenosis $(110 / 158,69.6 \%)$, shrinking lumen stenosis $(32 / 158$, $20.3 \%)$, and venous valve-related stenosis $(16 / 158,10.1 \%)$. In their analysis, the AVF stenosis pattern affected the outcome after PTA; the shrinking lumen morphology had a negative impact on primary patency (HR 2.05, 95\% CI $1.25-3.36$, and $p=0.005$ ), while the venous valve-related stenosis had a positive impact (HR 0.19, 95\% CI $0.04-0.79$, and $p=0.023$ ). To the authors' knowledge, there are yet to be recognized classification criteria for dysfunctional AVF stenosis lesions. Therefore, we adopted an intimal thickness of $0.6 \mathrm{~mm}$ on the stenotic site as the cutoff value according to previous research. The intimal thickness of human saphenous veins obtained during coronary artery bypass surgery was found to range from 18.80 to $241.3 \mu \mathrm{m}$ [19], and Hozumi et al. [20], using intravascular ultrasound in the great saphenous vein for aortocoronary bypass graft, showed a mean intimal thickness of $0.31 \pm 0.09 \mathrm{~mm} 1$ month after the operation and $0.65 \pm 0.08 \mathrm{~mm} 1$ year after the operation.

Under this method of classification, the primary patency rate at 6 and 12 months in the NIH group (Type I) in our cohort was lower than the results reported in the previous studies using drug-coated balloons (DCBs) but better than using standard PTA. Patane et al. [21] reported 2 types of DCBs compared with the standard PTA in the treatment of juxta-anastomotic stenosis of mature but dysfunctional AVFs; the primary patency rates were 81.8 and $84.1 \%$ in the DCB groups and $54.5 \%$ in the standard PTA group after 12 months. Irani et al. [22] reported in their randomized controlled trial (RCT) comparing DCB PTA (IN.PACT Admiral DCB, Invatec Medtronic) with conventional PTA for dysfunctional AVFs and AVGs that the primary patency in the DCB group was 81 and $51 \%$ after 6 and 12 months, while it was 61 and $34 \%$ in the standard PTA group. These reports indicate that dysfunctional AVFs and AVGs caused by lesions of intimal hyperplasia could benefit from the antiproliferative therapy by DCBs, rather than plain balloon angioplasty.

As for the non-NIH group, the 6-month and 1-year primary patency rates were not worse than those reports of the DCBs from previous RCTs [23-25]. Although it is less persuasive to compare the results from different tri- als, it indicated that PTA by high-pressure balloons alone without DCB may be enough for lesions not initiated by intimal hyperplasia. The univariable/multivariable Cox regression analysis also showed that the lesion type ( $p=$ 0.012 ) and anastomosis style (end-to-end anastomosis, $\mathrm{HR}=3.022, p=0.007$, CI: $1.328-6.764)$ were risk factors for lower primary and secondary patency after intervention of failed AVFs. In addition, previous PTA history was associated with lower secondary patency.

To some extent, our result explained why some RCTs were unable to show the superiority of DCB over plain old balloon angioplasty $[23,24,26]$. Whether the different pathological patterns are considered before comparing interventional treatments for dysfunction AVFs or not may affect the outcome. To improve the patency of intimal hyperplasia dominant lesions, routine balloon angioplasty is not as effective as DCBs. However, for non-NIH lesions, such as venous valve-related lesions, high-pressure balloons may be a first choice instead of DCBs, considering the current controversies about the safety of the latter. For lesions with instant elastic recoil that result in flow limitations after angioplasty or recurrent constriction by adventitia fibrosis, mechanical scaffolds (baremetal stent or covered stent) may be required. From this point of view, subgroup analysis based on preoperative lesion characteristics needs to be further investigated prospectively, rather than randomizing them without discrimination, which probably could better embody the advantage of different appliances.

This study has some limitations. First, this is a singlecenter study; due to its retrospective nature and relatively small number of patients, it is difficult to establish a cause and effect relationship and to generalize the findings to all dysfunctional AVF patients. It should also be mentioned that there is yet to be a consensus regarding the methodology of morphological classification of stenosis lesions under DU or digital subtraction angiography. The present criteria are conducive to classify the dysfunctional AVFs with clinical indicators, but further studies are warranted for the clinical validation and optimization of classification. Besides morphology patterns, other factors, such as the position of the AVFs (brachial or wrist), preoperative proximal flow volume, and the absolute area of the residual lesion, may also account for the patency rate after angioplasty. Some of the factors above were ascribed to Type III group, which caused a confounding bias, and thus need to be stratified and analyzed with a larger sample in the future.

In conclusion, our data indicated that the morphological patterns of dysfunctional AVF stenotic lesions are as- 
sociated with the outcome after interventional angioplasty. Non-NIH lesions tend to have a better prognosis, while the NIH-dominant type is a predictor of lower primary patency after high-pressure balloon dilatation. This study supports the concept that the morphological characteristics of stenotic lesions evaluated by DU need to be considered before treatment and individualized intervention strategy should be based on the histological properties of dysfunctional AVFs.

\section{Acknowledgment}

We thank the staff and participants from Huashan and Pudong Hospital for providing the clinical data for this research.

\section{Statement of Ethics}

This study was conducted according to the guidelines laid down in the Declaration of Helsinki, and all procedures involving human subjects were approved by Ethics Committee of Huashan Hospital. Written informed consent was taken from the participants.

\section{Conflict of Interest Statement}

The authors declare that they have no relevant financial interests.

\section{Author Contributions}

Research idea and study design: J.T., L.C., and W.Z.; data acquisition: W.Z. and L.C.; preoperative Doppler ultrasound: L.C. and Y.W.; PTA intervention: J.T., M.H., W.S., and B.Y.; statistical analysis: W.Z. and M.Z.; supervision or mentorship: J.T. and J.C.

\section{Funding Sources}

This work was supported by the National Natural Science Foundation of China (No. 51701041); Committee of Shanghai Science and Technology (No. 17DZ2200200); Program for Medical Key Department of Shanghai (ZK2019A10); and the Outstanding Clinical Discipline Project of Shanghai Pudong (PWYgy-2018-08).

\section{References}

1 Zhang L, Zhao MH, Zuo L, Wang Y, Yu F, Zhang $\mathrm{H}$, et al. China Kidney Disease Network (CK-NET) 2015 annual data report. Kidney Int Suppl (2011). 2019;9(1):e1-81.

2 Zhang L, Zuo L. Current burden of end-stage kidney disease and its future trend in China. Clin Nephrol. 2016;86(13):27-8.

3 Lok CE, Huber TS, Lee T, Shenoy S, Yevzlin AS, Abreo K, et al. KDOQI clinical practice guideline for vascular access: 2019 update. Am J Kidney Dis. 2020;75(4Suppl 2):S1-164.

4 Rothuizen TC, Wong C, Quax PH, van Zonneveld AJ, Rabelink TJ, Rotmans JI. Arteriovenous access failure: more than just intimal hyperplasia? Nephrol Dial Transplant. 2013;28(5):1085-92.

5 Roy-Chaudhury P, Sukhatme VP, Cheung AK. Hemodialysis vascular access dysfunction: a cellular and molecular viewpoint. J Am Soc Nephrol. 2006;17(4):1112-27.

6 Cunnane CV, Cunnane EM, Walsh MT. A review of the hemodynamic factors believed to contribute to vascular access dysfunction. Cardiovasc Eng Technol. 2017;8(3):280-94.

7 Duque JC, Tabbara M, Martinez L, Cardona J, Vazquez-Padron RI, Salman LH. Dialysis arteriovenous fistula failure and angioplasty: intimal hyperplasia and other causes of access failure. Am J Kidney Dis. 2017;69(1):147-51.

Lesion Type and AVF Patency Rate after PTA
8 Allon M, Litovsky S, Young CJ, Deierhoi MH, Goodman J, Hanaway M, et al. Medial fibrosis, vascular calcification, intimal hyperplasia, and arteriovenous fistula maturation. Am J Kidney Dis. 2011;58(3):437-43.

9 Simone S, Loverre A, Cariello M, Divella C, Castellano G, Gesualdo L, et al. Arteriovenous fistula stenosis in hemodialysis patients is characterized by an increased adventitial fibrosis. J Nephrol. 2014;27(5):555-62.

10 Hemodialysis Adequacy 2006 Work Group. Clinical practice guidelines for hemodialysis adequacy, update 2006. Am J Kidney Dis. 2006;48(Suppl 1):S2-90.

11 Allon M, Litovsky SH, Zhang Y, Le H, Cheung AK, Shiu YT. Association of preexisting arterial intimal hyperplasia with arteriovenous fistula outcomes. Clin J Am Soc Nephrol. 2018;13(9):1358-63.

12 Quencer KB, Arici M. Arteriovenous fistulas and their characteristic sites of stenosis. AJR Am J Roentgenol. 2015;205(4):726-34.

13 Murakami M, Miyamoto M, Suemitsu K. Percutaneous transluminal angioplasty in Japan: five-center investigation. J Vasc Access. 2015; 16(Suppl 10):S38-42.

14 Skorecki K, Chertow G, Marsden P, Taal M, Yu A. Brenner and Rector's the kidney. 10th ed.; 2015.

15 Meier GH. Hemodialysis access: failing and thrombosed. In: Cronenwett JL, Johnston KW, editors. Rutherford's vascular surgery. 8th ed. Philadelphia: Saunders Elsevier; 2014.
16 Tabbara M, Duque JC, Martinez L, Escobar $\mathrm{LA}, \mathrm{Wu} \mathrm{W}, \mathrm{Pan} \mathrm{Y}$, et al. Pre-existing and postoperative intimal hyperplasia and arteriovenous fistula outcomes. Am J Kidney Dis. 2016;68(3):455-64.

17 Yamamoto Y, Nakamura J, Nakayama Y, Hino H, Kobayashi H, Sugiura T. Relationship between the outcomes of stent placement and the properties of arteriovenous graft outflow vein stenotic lesions. J Vasc Access. 2012; 13(4):426-31.

18 Suemitsu K, Shiraki T, Iida O, Kobayashi H, Matsuoka Y, Izumi M, et al. Impact of lesion morphology on durability after angioplasty of failed arteriovenous fistulas in hemodialysis patients. J Endovasc Ther. 2018;25(5):649-54.

19 Li FD, Sexton KW, Hocking KM, Osgood MJ, Eagle S, Cheung-Flynn J, et al. Intimal thickness associated with endothelial dysfunction in human vein grafts. J Surg Res. 2013;180(1): e55-62.

20 Hozumi T, Yoshikawa J, Yoshida K, Akasaka $\mathrm{T}$, Takagi T, Honda $\mathrm{Y}$, et al. Use of intravascular ultrasound for in vivo assessment of changes in intimal thickness of angiographically normal saphenous vein grafts one year after aortocoronary bypass surgery. Heart. 1996;76(4):317-20 
21 Patane D, Failla G, Coniglio G, Russo G, Morale W, Seminara G, et al. Treatment of juxtaanastomotic stenoses for failing distal radiocephalic arteriovenous fistulas: drug-coated balloons versus angioplasty. J Vasc Access. 2019;20(2):209-16.

22 Irani FG, Teo T, Tay KH, Yin WH, Win HH, Gogna A, et al. Hemodialysis Arteriovenous fistula and graft stenoses: randomized trial comparing drug-eluting balloon angioplasty with conventional angioplasty. Radiology. 2018;289(1):238-47.
23 Maleux G, Vander MW, Henroteaux D, Laenen A, Cornelissen S, Claes K, et al. Multicenter, randomized trial of conventional balloon angioplasty versus paclitaxel-coated balloon angioplasty for the treatment of dysfunctioning autologous dialysis fistulae. J Vasc Interv Radiol. 2018;29(4):470-475. e3.

24 Trerotola SO, Lawson J, Roy-Chaudhury P, Saad TF. Drug coated balloon angioplasty in failing AV fistulas: a randomized controlled trial. Clin J Am Soc Nephrol. 2018;13(8): 1215-24.
25 Khawaja AZ, Cassidy DB, Al SJ, McGrogan DG, Inston NG, Jones RG. Systematic review of drug eluting balloon angioplasty for arteriovenous haemodialysis access stenosis. J Vasc Access. 2016;17(2):103-10.

26 Bjorkman P, Weselius EM, Kokkonen T, Rauta V, Alback A, Venermo M. Drug-coated versus plain balloon angioplasty in arteriovenous fistulas: a randomized, controlled study with 1-year follow-up (The Drecorest IiStudy). Scand J Surg. 2019;108(1):61-6. 